http://jaet.journals.ekb.eg

\title{
DESIGN AND IMPLEMENTATION OF A PROMISING OPTICAL SUBSYSTEM WITH A SKY CAMERA FOR LASER WARNING SYSTEMS
}

\author{
Abdalrhman Adel mohamed ${ }^{1, *}$, Moustafa Ahmed ${ }^{2}$ \\ Mohamed Mabrouk ${ }^{3}$, Hesham F. A. Hamed ${ }^{4}$ \\ ${ }^{I}$ Electrical and communication engineering, Faculty of Engineering, minia University, Egypt \\ ${ }^{2}$ Department of physiscs, Faculty of science, Minia University, Egypt \\ ${ }^{3}$ Electrical and communication engineering, Faculty of Engineering, Alexandria University, Egypt \\ ${ }^{4}$ Faculty of Engineering, Minia University, Egypt \& Faculty of Engineering, Egyptian-Russian University, Cairo, Egypt \\ labdou40105@gmail.com_2mostafa.farghal@mu.edu.e_3mmabr084@uottawa.ca ${ }^{4}$ hfah66@yahoo.com
}

\section{ABSTRACT}

In the last few decades, a Significant advance in the laser detection techniques have occurred in a civilian application like optical fiber communication system, lidar system and now satellite communication in the erospace and a military application, one of the most applications in military is the laser guided missiles and bombs encountered high development and formed high threat for strategic and special targets. The development of a laser warning system (LWS) is essential to detect the laser guided weapons attack. A classic LWS is composed of an optical subsystem, laser detector, and processing unit. The design of a LWS is constrained by the cost, optical system size, detector type, and the required processing operations. In this paper, a 15-degree field of view optical subsystem, diffraction grating, and a sky camera is used to reduce the whole system size, reduce the cost, and decrease the number of false alarms initiated by the dark current on the classical detectors. The optical subsystem is designed to shape the laser spot diameter to be $350 \mu \mathrm{m}$ to avoid detection saturation. The design and fabrication of the optical system guaranteed a signal-tonoise ratio up to $175 \mathrm{~dB}$ in a clear atmospheric condition. This LWS outperforms the state-ofart LWSs in laser guided weapon detection.

Keywords: (laser; LWS; guided missile; sky camera; optical system)

\section{INTRODUCTION}

During the last century, different types of bombs have been considered in many wars, especially, world-war-II [1]. After that, the classical bombs were developed to be smart. The missile/bomb guidance methods are: millimetric waves, television, infrared, and laser [2]. Missile and bomb laser guidance method outperforms the other methods in

Received:4 October, 2020, Accepted:4 January, 2021 high precision degree of hitting a destroying the target [3]. Many methods were developed to counter the laser guided missiles. The first step is to detect the threat by detecting laser rangefinders or laser target designators. One of the methods to detect the laser threats is to use a laser warning system (LSW) [4]. A laser warning system consists of a detection head that includes an optical subsystem, sensor detection and a processing unit [5]. 


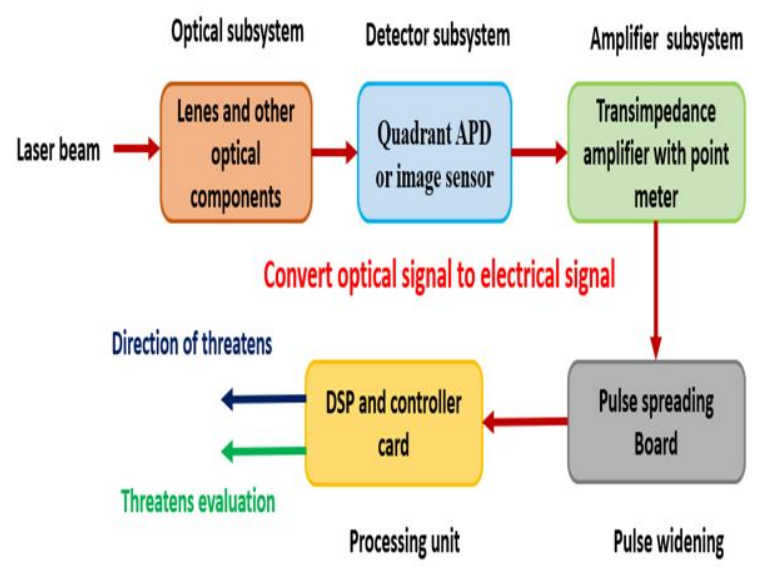

Figure.1. The classical LWS general block diagram

The incident laser beam is focused and directed by the optical subsystem and passed to a detection subsystem [6]. Figure 1 shows the classical LWS general block diagram. The laser beam incidents on the lenses of the optical system and then gathered, it focused on a photo detector such as the quadrant avalanche photo diode (APD) [7]. The output of the detector is converted from optical to electrical signal and directed to a trans-impedance amplifier with point meter to adapt the amplifier gain to give a specific output value. Then the laser pulse is widened in a preprocessing step and sent to the processing unit where a threshold is applied for detection process [8]. Figure 2 shows a LWS detecting a laser pulse at $108^{\circ}$ from the north direction.LWS is designed to be functional in different battlefield situations [9]. The design shall be simple to be implemented and the cost shall be reduced without affecting the system overall technical characteristics. LWS is implemented with an array of sensors and light control filter [10]. An array of sensors is used to detect the laser source direction and the filter is used to reject the frequencies out of the frequency band for the laser source. However, the direction of the laser source can be detected using the ability of the optical system to change the field of view or rotate the optical system on a rotating pedestal [11]. Using an array of sensors is an expensive choice. A sky camera may be used to reduce the cost of the LWS.
The design and implementation of the optical system is constrained with the LWS overall size. An optical system composed of six lenses was used to implement LWS [12]. However, the size and the number of the lenses can be reduced to reduce the overall size. In this paper, LWS is designed and implemented using sky camera, small size optical subsystem, diffraction grating, and processing unit. We develop a new design of a $0.4-1.1 \mu \mathrm{m}$ optical subsystem for use in the detector head of LWSs. We use an array of three lenses for appropriate gathering of the incident laser radiation. The lenses are attached to a sinusoidal diffraction grating to detect the wavelengths of incident light and separate the back ground noise due to sunlight rays. The laser beam is then captured and analysed using a sky camera. The laser spot diameter focused on the camera is smaller than $350 \mu \mathrm{m}$ in the worst case. The field of view is 15 degrees and SNR is better than $170 \mathrm{~dB}$. The demonstrated laser detector operates in distances very far from laser sources and in hazy conditions. The novel system has low cost, reduced size, and high received signal-to-noise ratio (SNR). The paper is organized as follows: section 2 presents methodology and optical subsystem design, section 3 presents the implementation of the LWS, section 4 presents the results, and section 5 presents conclusion.

\section{METHODOLOGY OF THE OPTICAL SUBSYSTEM DESIGN}

An optical warning detection system should be able to distinguish pulsed laser sources with very narrow pulse width as well as continuous signals with wavelengths ranging between 0.4 and $1.1 \mu \mathrm{m}$. Detection of these signals at far ranges is difficult, and designing an optical array to gather this wide range of wavelengths is effortful. we propose the design of an optical subsystem capable of gathering laser radiation on the detector and distinguishing radiations with 10 degrees over the horizon. So, the field 
Of view (FOV) of the proposed optical subsystem is 15 degrees. This is optimum to detect the incident laser in far field practically from our experience. The optical subsystem is divided into five main parts; namely, a lens array to gather the incident laser radiation, a diffraction grating to detect the frequencies of the incident light and separate the laser components from background noise, a sky camera sensor, processing unit and an angle indicator using optical decoder. The implemented system costs $1500 \$$ using on shelf components. Compared to the subsystem proposed by Najad et al. [13], this route is advantageous in determining the wavelength and incident angle of the incident laser radiation simultaneously by a simple optical design and low cost. In addition, the overall size of the optical system is reduced using three lenses instead of the six lenses utilized by [13].

\section{Design of Three-Lenses System for Gathering Laser Radiation}

The array of lenses works to gather the incident laser light and path through it to the sky camera detector. A two-dimensional view of the lens array is shown in Fig. 2. The front lens is made of sk 2 and with thickness $8.44 \mathrm{~mm}$ and the front lens is made of sk2 with thickness of $8.44 \mathrm{~mm}$ and with front and back radii of $118.9 \mathrm{~mm}$ and $91.961 \mathrm{~mm}$ respectively. The lens has two spherical surfaces; both are convex but with different radii of curvature.

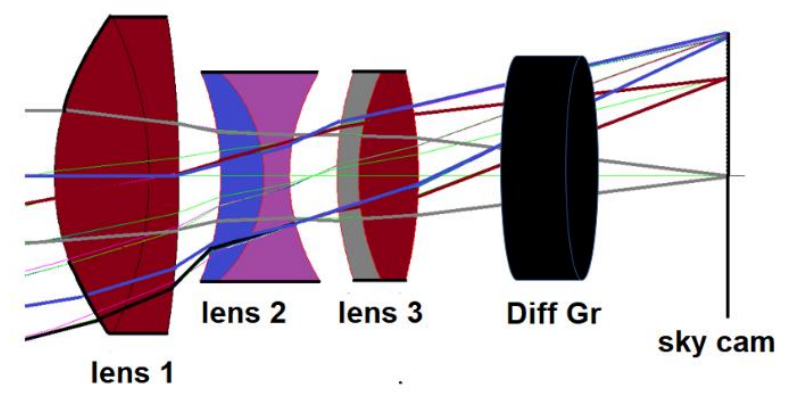

Figure.2. 2-D schematic of the proposed optical

It is equivalent to the aspheric lens but provides more reduction of the monochromatic aberration; this lens setup can be used to transfer a collimated beam from an incident point light on the detector similarly like a spherical lens. The middle lens is a biconcave lens made of sk16 with thickness of $6.34 \mathrm{~mm}$ and with front and back radii of $52.3 \mathrm{~mm}$ and $32.651 \mathrm{~mm}$ respectively. This is able to reduce both the coma and spherical aberration in addition to the astigmatism. Furthermore, this lens provides the advantage of aspheric lenses in qualifying the degree of resolution. The last lens is made of sk16 with thickness $2.76 \mathrm{~mm}$ and with front and back radii of $48.4 \mathrm{~mm}$ and $15.956 \mathrm{~mm}$ respectively. Since this is the final lens facing the CCD of the camera, it is a biconvex lens which functions in overcoming aberration of the formed image and the distortion field curvature. The separating distance between the objective and middle lenses is $8.301 \mathrm{~mm}$, while it is $40.66 \mathrm{~mm}$ between the middle and final lenses. These design parameters were optimized according to the theories of optical systems and the associated image aberration in Ref. [15]. The optical materials of the lenses and antireflection coatings are chosen to achieve the highest optical transmission and reduce the absorption, and reflection. These optical characteristics of the lens system are calculated using the computer software Win Lens and Zemax.

\section{Diffraction grating and Sky Camera Detector}

The above designed array of lenses is attached to a diffraction grating. The diffraction grating is used in LWS to measure the incident laser wavelength and adjust the incident angle [16]. This grating is advantageous in conquering the overlap among different wavelengths and different diffraction orders [16-17], simplifying the abstraction algorithm of the wavelength and incoming angle, and concentrating the incident laser beam on the detector [17]. The laser beam impinges on the grating, then diffracts and focuses by the lens on the sky camera. Then, the diffraction pattern is transformed into an electrical signal by the sky camera. This electrical signal is processed 
By a high-speed digital signal processor (DSP) of type LSA2136, and finally the information of the wavelength and incident angle are obtained.

\section{Spot size, aberration, and SNR calculations}

The spot size of the laser beam is determined assuming propagation of an ideal Gaussian beam. The intensity distribution in a Gaussian beam is represented by a bundle of rays in which each ray has a random pointing error. The ray bundle is allowed to pass through proposed optical system the usual geometrical optics formulae and it is shown that the position of the focused beam waist agrees exactly with the usual Gaussian mode analysis in [18], the spot size of the beam along the propagation $\mathrm{z}$ direction is determined by:

$$
W(z)=W o \sqrt{1+\left(\frac{\lambda Z}{\pi W_{0}^{2}}\right)^{2}}
$$

Where the $W(z)$ is the spot radius in an arbitraryz, $W_{0}$ is the minimum spot diameter and $\lambda$ is the wavelength of the radiation. Figure 4(a) far field in a clear conditions and 4(b) far field in a hazy conditions

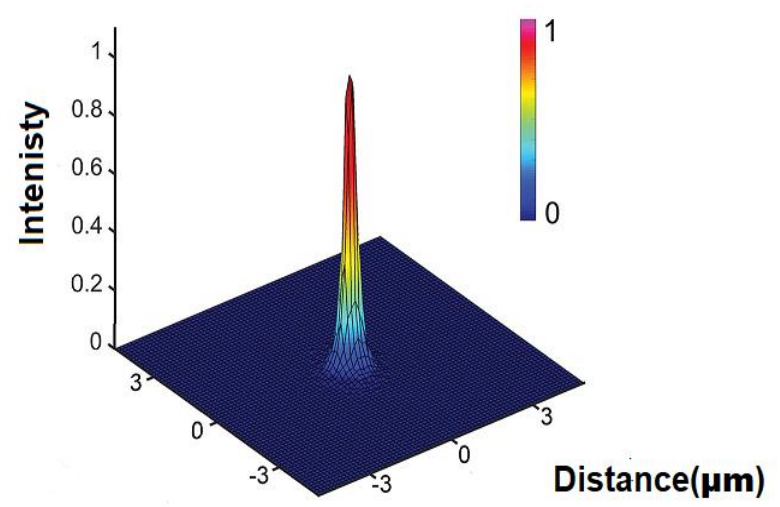

(a)

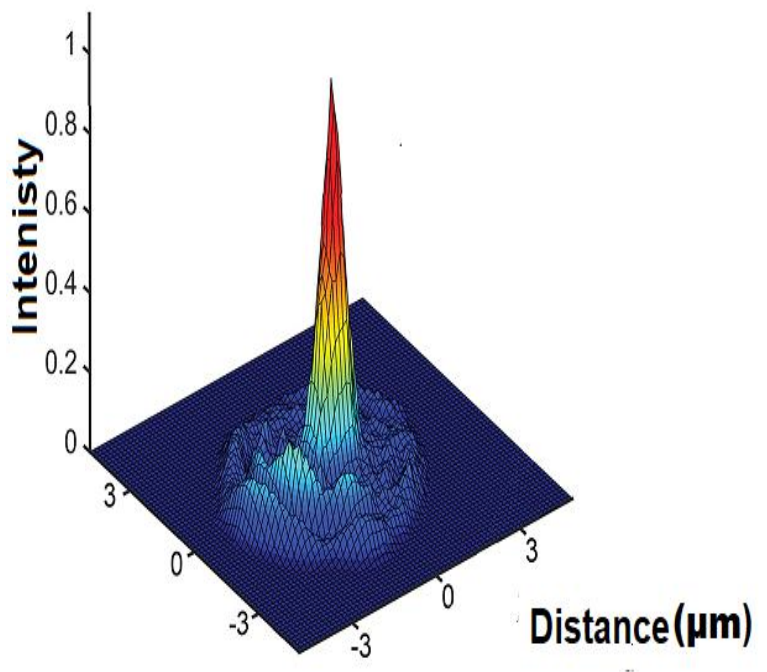

(b)

Figure.3. Laser beam profiling (a) in a far field clear (b) in a far field hazy.

Another important parameter, which should be considered in the design of the optical subsystem, is the aberration of the proposed optical array. Although the aberrations include spherical, coma, astigmatism, achromatic and distortion, which are called "five Seidel's aberrations" [19], the effective ones for the present lens array are the achromatic, coma and spherical aberrations because the other aberrations are addressed by sky camera. Large values of these three types of aberration mean that the noise is high and the sky camera sensor cannot define the laser wavelength. Figure 5 shows the seidel's chart of the lens array using Win Lens and Zemax software. This chart displays the aberration parameters to each surface of the three lenses separately. The measured values of the total aberrations for the present lenses array are chromatic aberration of- $0.45 \mathrm{~mm}$, coma aberration of $-0.29 \mathrm{~mm}$, and spherical aberration of $-1.65 \mathrm{~mm}$. These values are optimum to achieve qualified detection of the incident laser beam [20]. 


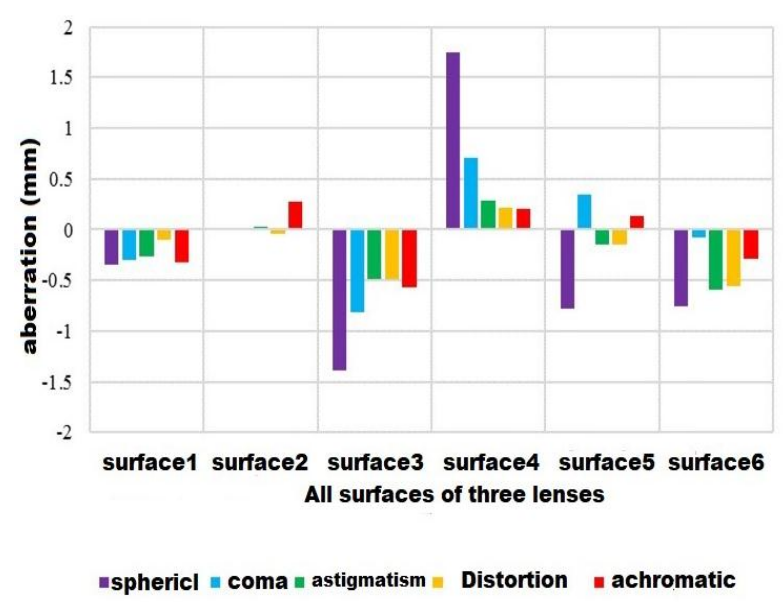

Figure .4. Seidel's chart to each surface separately parameters

The Seidel aberration function is given by the following equations [19]:

$$
\varepsilon_{x}=S_{1} \rho^{3} \sin \varphi+S_{2} Y \rho^{2} \sin 2 \varphi+P Y^{2} \rho \sin \varphi
$$

$$
\begin{aligned}
& \varepsilon_{y}=S_{1} \rho^{3} \cos \varphi+S_{2} Y \rho^{2}(2+\cos 2 \varphi)+ \\
& \left(S_{3}+P\right) Y^{2} \rho \cos \varphi+S_{5} Y^{3}
\end{aligned}
$$

where $\varepsilon_{x}$ is the Seidel aberration in the $x$ axis direction, $\varepsilon_{y}$ is the Seidel aberration in the $y$ axis direction, $S_{1}$ is the spherical aberration coefficient, $S_{2}$ is the Coma coefficient, $S_{3}$ is the astigmatism coefficient, $P$ is the Petzval sum coefficient, $S_{5}$ is the distortion coefficient, $Y$ is the ideal image height on the image plane, $\varphi$ is the ray position angle at the exist pupil, and $\rho$ is the ray height at the exist pupil. These chart yield information on the best results of minimum aberration values of the present proposed system. Another important parameter that should be examined in the optical array is the signal-to-noise ratio (SNR), which can be calculated according to the following equation [21].

$$
S N R=\frac{V s}{V n}=\frac{4 P o D_{\lambda}^{*}}{\pi \theta^{2} R^{2}} \sqrt{\frac{A_{d}}{\Delta F}} \tau_{a} \tau_{o}
$$

Where $V S$ is the maximum voltage of the detector, $V_{n}$ is the environmental noise signal
Voltage, $D_{\lambda}^{*}$ is a detective parameter at a particular wavelength, $\tau_{a}$ is the atmospheric attenuation for the laser, $R$ is distance from the laser source to the detector, $\tau_{0}$ is the optical transmission coefficient, $\theta$ is laser's divergence in radian, $A_{d}$ is the laser spot's area on the detector, $\Delta F$ is the detector's bandwidth the proposed system and $P_{0}$ is a peak power laser source .LWS is a military application

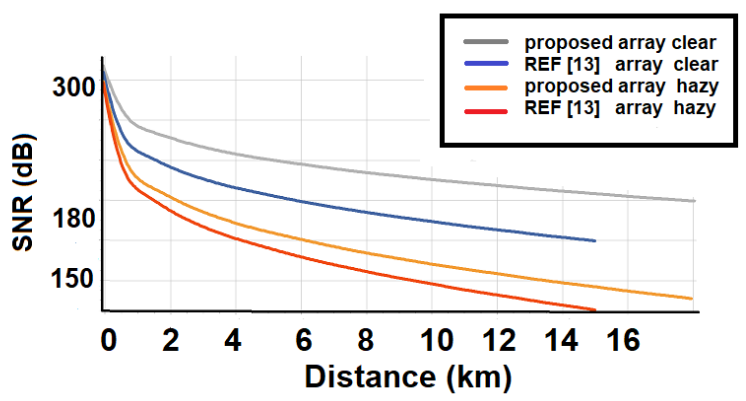

Figure.5. SNR for the proposed optical array in clear and hazy atmospheric conditions compared to the work in [13]

Its work in very hard battle field conditions, so the SNR of the laser detector received signal must be as high as possible to detect laser sources at high ranges [21]. Figure 5plots $S N R$ as a function of distance $R$ and the results are compared with those in Ref. [13]. SNR in clear atmospheric conditions is higher than that in hazy atmospheric conditions by more than 30 $\mathrm{dB}$. The SNR of the proposed optical system in both conditions are higher than that in [13] because the detective parameter $D_{\lambda}^{*}$ and the detector effective area are higher when using the sky camera detector. The active detective area is 20 times more than in [13]. Although the SNR of the proposed system is enhanced, SNR is degraded more in the proposed system in hazy atmospheric conditions

\section{IMPLEMENTATION OF THE LASER WARNING HEAD}

This section is concerned with implementation of the designed optical 
Subsystem. First, the lens array was manufactured according to the aforementioned specifications. The lens array is designed in three dimensions by the 3D model of the Optocad program. The designed $3 \mathrm{D}$ model of the lens array is shown in Fig. 6 . The lens arrays were placed in an aluminum chassis closed tightly and charged with nitrogen gas to avoid moisture from water vapor.

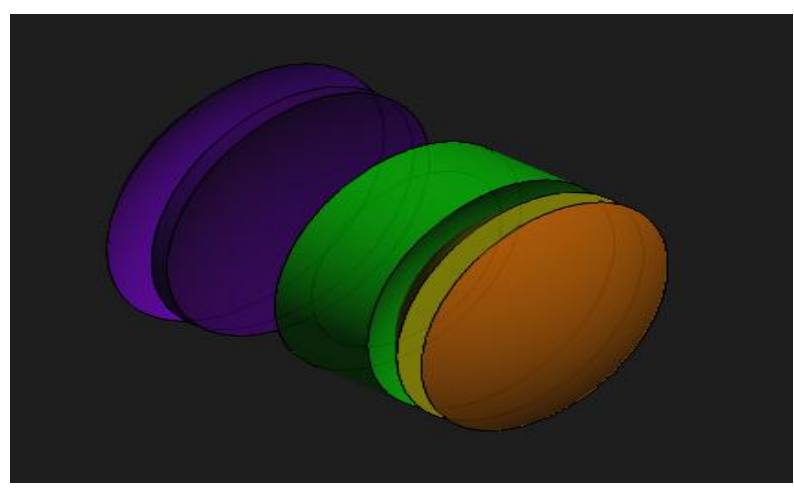

Figure.6. 3D view of the glass lenses

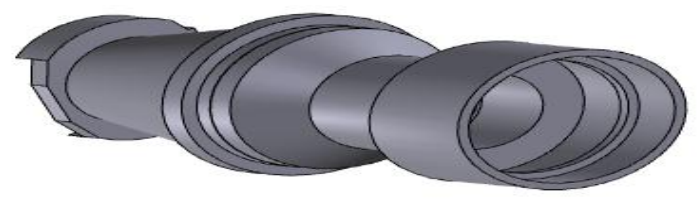

Figure.7. Layout of the implemented lens array.

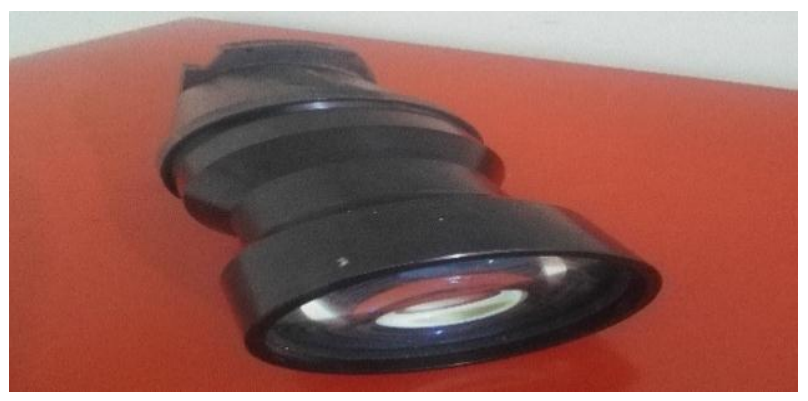

Figure.8. Layout of the chassis of optical proposed

- The length of the chassis is $25 \mathrm{~cm}$, the front diameter is $15.5 \mathrm{~cm}$, and the chassis diameter of the middle is $8.5 \mathrm{~cm}$ as shown in Fig. 7. A layout of the implemented lens array is shown in Fig. 8.

\section{RESULTS}

In our experiments, we used three laser sources with wavelength $\lambda=532 \mathrm{~nm}, 808 \mathrm{~nm}$ and $1064 \mathrm{~nm}$. The optical subsystem was carried on a pan-tilt motor to enable $360^{\circ}$ scanning of the field of view. The optical system is attached with an optical digital encoder which is connected to a computer to measure the angle of incidence of the laser radiation relative to the axis of the lens array. Incident angle of $\theta=90^{\circ}$ corresponds to the case of on-axis incidence to the detection head. Fig. 9 plots the spectrum of the incident laser in existence of sun light as detected before incidence on the sky camera by a digital high-speed DSP spectrometer with a digital oscilloscope (model Aglint 86100A).Fig.10 plots the laser real measurement formed by spectrometer after using sky camera only, this figure shows the background noise from sun, laser source $532 \mathrm{~nm}$ and multi-mode laser .The spectrum data were exported serially to the computer and plotted using special software. The figure indicates noisy spectrum and the laser spectrum cannot be distinguished. On the other hand, Fig. 11 plots the spectrum after passing through the proposed optical subsystem ,diffraction grating and then recorded by the sky camera for laser emitted at wavelength $\lambda=532 \mathrm{~nm}$. In the other spikes we utilized other laser sources incident off axis on a proposed system with a background noise to be sure the proposed system can be detected and identify the main laser sources $532 \mathrm{~nm}, 808 \mathrm{~nm}$ and $1064 \mathrm{~nm}$ on axis by a higher intensity than other laser sources off axis Separates from back ground noise. The laser beam is directed one time in the on-axis direction of the optical head and in another time with an angle of off-axis. It is clear that the shown spectrum corresponds to the laser source only. That is, our detection system is able to remove the background noise with the laser beam. Fig.12 show the real images were captured by proposed system and identify the angle of laser beam $\left(90^{\circ}\right)$ on axis and $\left(58^{\circ}\right)$ off axis and 


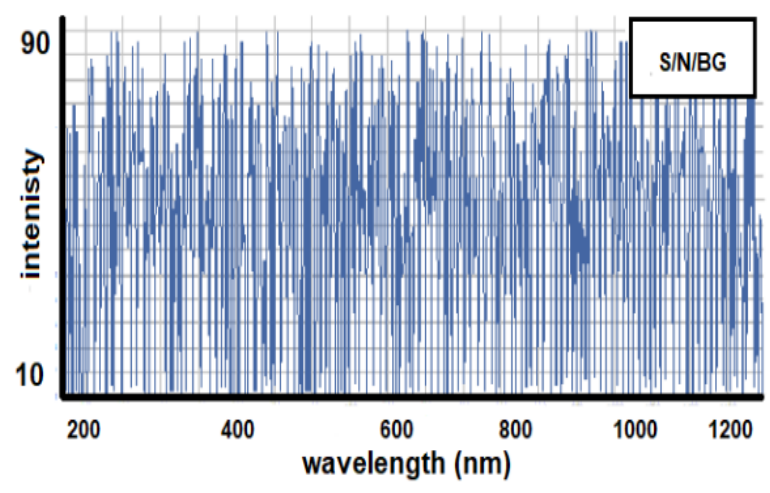

Figure.9. the spectrum of the incident laser in existence of sun light

Figures 13 to 17 shows the spectrum of incident laser beams at wavelengths of $\lambda=808$ and $1064 \mathrm{~nm}$, respectively. The system was able to detect the laser beam at these wavelengths.

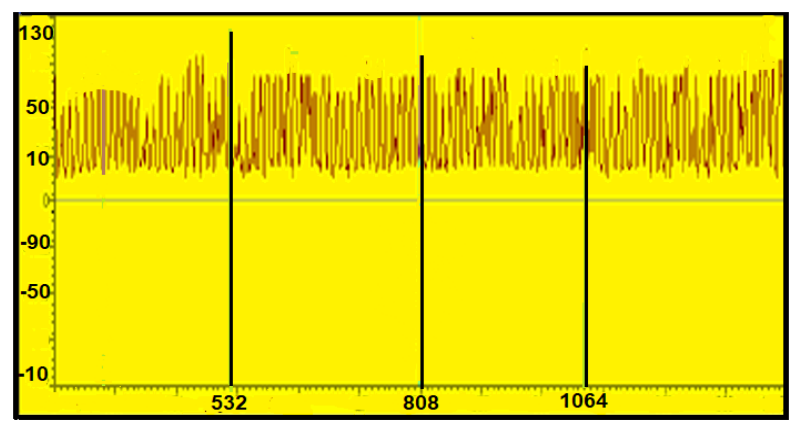

Figure.10.532 nm laser real measurement formed by Spectrometer before using proposed system.

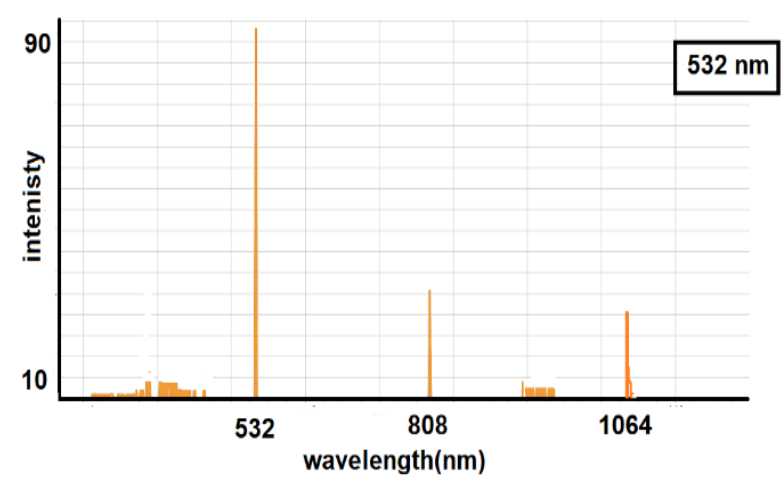

Figure.11. the spectrum after passing through the diffraction grating and then recorded by the sky camera for laser emitted at wavelength $\lambda=532 \mathrm{~nm}$
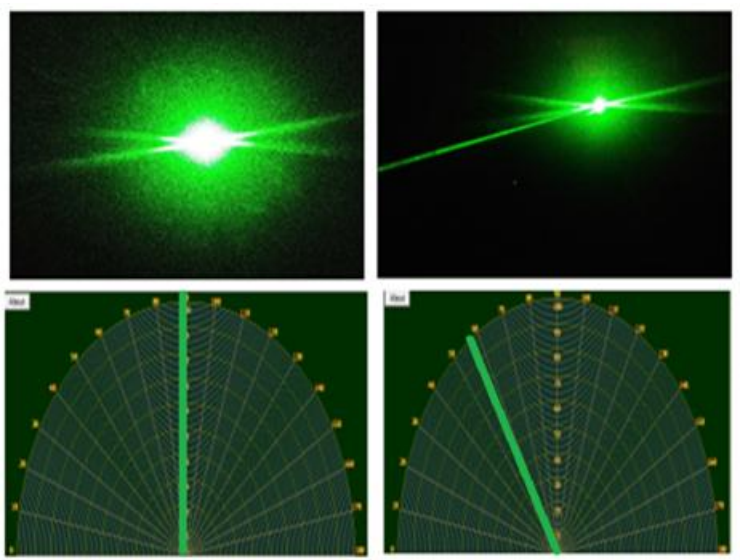

90

deg

58

Figure.12. the real images were captured by proposed system and identify the angle of laser beam $\left(90^{\circ}\right)$ on axis And $\left(58^{\circ}\right)$ off axis

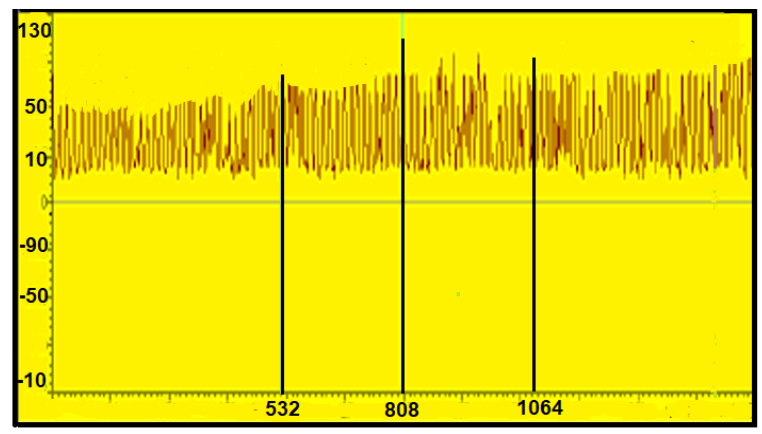

Figure $13.808 \mathrm{~nm}$ laser real measurement formed by spectrometer before using proposed system

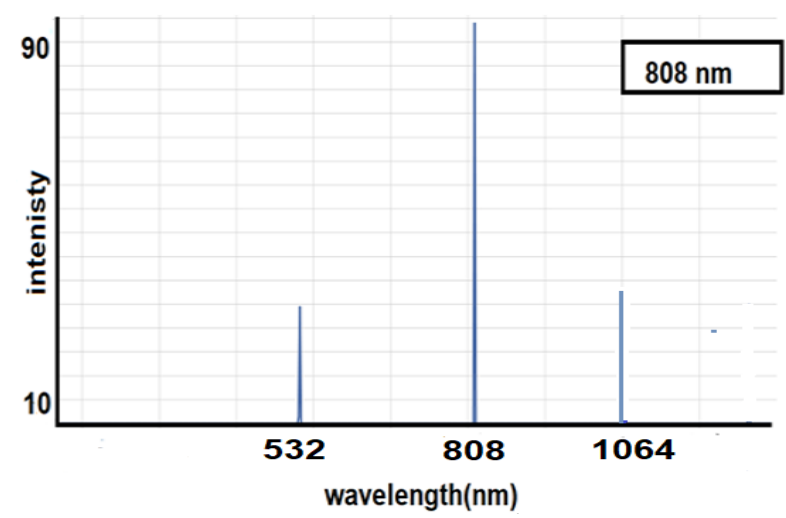

Figure.14. the spectrum after passing through the diffraction grating and then recorded by the sky camera for laser emitted at wavelength $\lambda=808 \mathrm{~nm}$ 

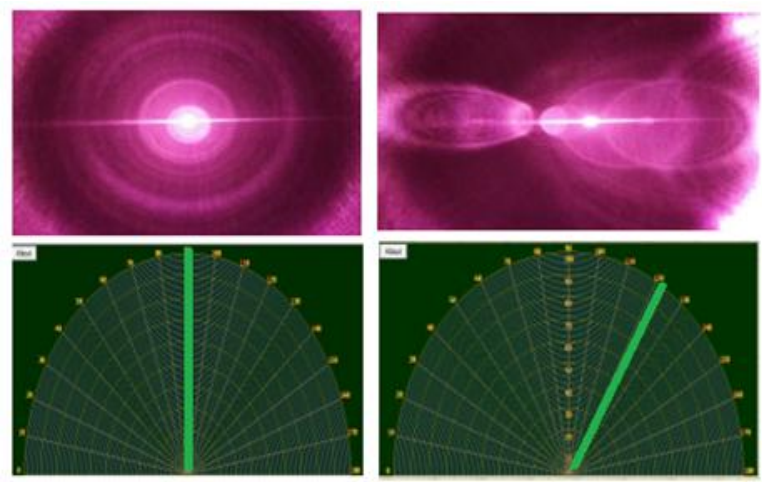

90

deg

122

Figure.15. The real images were captured by the proposed system and identify the angle of laser beam $\left(90^{\circ}\right)$ on axis and $\left(122^{\circ}\right)$ off axis

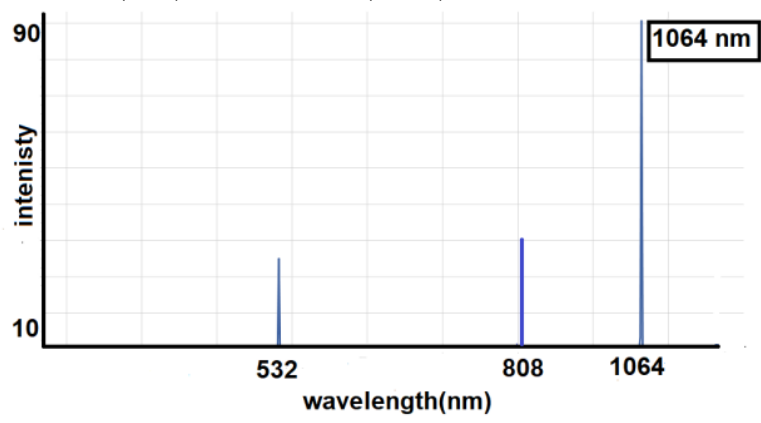

Figure 16.The spectrum after passing through The diffraction grating and then recorded by the sky
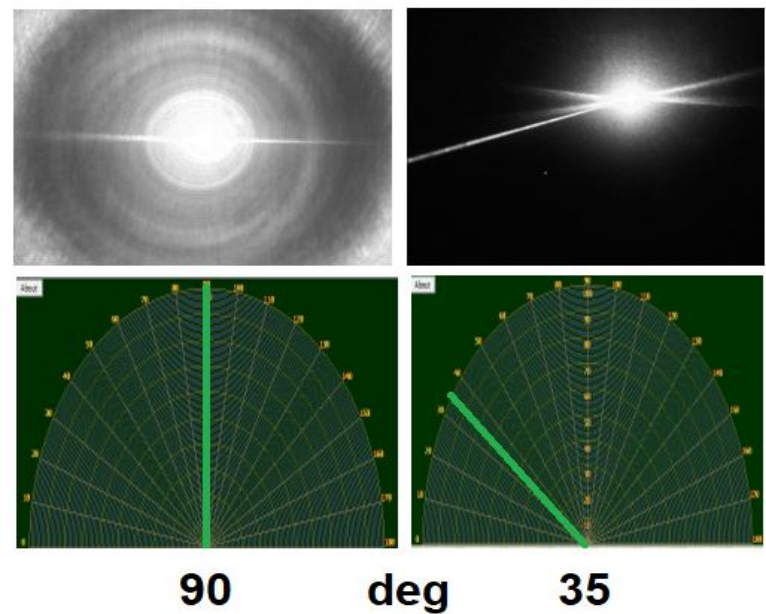

\section{deg}

\section{5}

camera for laser emitted at wavelength $\lambda=1064 \mathrm{~nm}$

Figure.17. the real image captured by the proposed system and identifies the angles of laser beam $\left(90^{\circ}\right)$ and $\left(35^{\circ}\right)$

\section{Conclusions}

A laser warning system based on new optical subsystem, sky camera, and diffraction grating is designed and implemented. The optical subsystem is composed of an array of three lenses attached to a diffraction grating and utilized a sky camera sensor and a high-speed DSP spectrometer to detect the laser source wavelength and the angle of arrival. The system has low cost and the results showed that it can detect laser sources at wavelengths $532 \mathrm{~nm}, 808 \mathrm{~nm}$, and $1064 \mathrm{~nm}$. Using this result; we can identify the incident laser from background noise. There is no false alarm rate because it depends on a real image of the incident laser spot size, as we can see a real image of laser incident by special monitor. Compared to the work in [14] SNR $160 \mathrm{~dB}$, the proposed system achieve SNR up to $175 \mathrm{~dB}$ in a clear conditions.

\section{REFERENCES}

[1] G. Richey, "Guided missiles: A review of their history and design features," Students' Quarterly Journal, vol. 38, no. 151, pp. 139-145, 1968.

[2]

A. S. Gad and M. S. Aly, "Smart bomb's guidance loop design," in 8th international conference on aerospace sciences \& aviation technology, Cairo, 1999.

[3] M. Li, J. Yin , J. Wang and J. Xu, "Rapid design of guidance and control loop for laser guided bomb," in 32nd Chinese Control Conference, Xi'an, 2013. 
[4] D. R. Jungwirth, "Laser detection and warning system". Europe Patent EP2752681A1, 2282018.

[5] S. Kumar, S. Prakash, A. K. Maini, V. Patil and R. Sharma, "DESIGN OF A LASER-WARNING SYSTEM USING AN ARRAY OF DISCRETE PHOTODIODESPART I," JOURNAL OF BATTLEFIELD TECHNOLOGY, vol. 14, no. 1, pp. 13-16, 2011.

A. K. Maini, Lasers and optoelectronics: fundamentals, devices and applications, United Kingdom: John Wiley \& Sons, 2013.

[7] Fotona, "Laser warning system," Fotona choose perfection, $\begin{array}{lll}7 & 10 & 2020 .\end{array}$ [Online].

Available:https://www.fotona.com/en/product s/2468/lird-4b/.

[8] J. Che, J. Zhang, H. Wang, and B. Cheng, "Error Analysis of Angular Resolution for Direct Intercepting Measurement Laser Warning Equipment," in Proc. SPIE 10141, Selected Papers of the Chinese Society for Optical Engineering Conferences held July 2016, vol. 10141, pp. 101410U1-6, 2016.

[9] M. Jurba, E. Popescu, S. Cojocaru, D. Guiman and D. Stroe, "Extended spectral range laser receiver," Optoelectronics and Advanced Materials-Rapid Communication, Vol. 6, No. 11, pp. 1181-1184, Nov. 2012

[10] J. Dubois and F. Reid, "Detecting laser sources on the battlefield," Photonics North 2007, vol. 67962F, 2007
[11] Z. Jilong, T. Erming and W. Zhibin, "Research on Laser Warning Receiver Based on Sinusoidal Transmission Grating and High-Speed DSPs," WSEAS Transactions on Circuits and Systems, vol. 5, no. 8, pp. 1-6, 2006.

[12] D. M. Benton, "Laser detection using liquid crystal polarization modulators," Optical Engineering, vol. 59, no. 6, 2020.

[13] M. Nejad, H. Arab and N. R. Sheshkelani, "Analysis of New Laser Warning Technologies to Propose a New Optical Subsystem," Iranian Journal of Electrical and Electronic Engineering, vol. 14, no. 3, pp. 213-221, 2018.

[14] Su, Ping, et al. "Image acquisition and processing of a laser warning system based on planar array CCD." Laser Technology 37.3 (2013): 394-399.

[15] O. Shulika and I. Sukhoivanov, Advanced Lasers: Laser Physics and Technology for Applied and Fundamental Science, Florida: Springer, 2015.

[16] F. Huang, R. Klette and K. Scheibe, Panoramic Imaging Sensor Line Cameras and Laser Range Finders, Germany: Wiley, 2008.

[17] J. Bentley and C. Olson, Field Guide to Lens Design, SPIE press book, 2012.

[18] S. G. Srinivasa, "Models and algorithms for vision through the atmosphere," Columbia University, New York, 2004. 
Vol. 41, No.2. July 2022

[19] Jungwirth, Douglas R. "Laser detection and warning system." U.S. Patent No. 9,134,174. 15 Sep. 2015.

[20] Min, Kyoung Moo, et al. "System and method of providing warning to pedestrian using laser beam." U.S. Patent No. 9,024,740. 5 May 2015.

[21] J. R. Wootton, Laser Warning Systems and Methods. US Patent No. US20030234349A1, Dec. 2003.S. 